PROCEEDINGS OF THE

AMERICAN MATHEMATICAL SOCIETY

Volume 137, Number 9, September 2009, Pages 2835-2841

S 0002-9939(09)09858-X

Article electronically published on February 16, 2009

\title{
RESONANCE OF BASIS-CONJUGATING AUTOMORPHISM GROUPS
}

\author{
DANIEL C. COHEN
}

(Communicated by Daniel Ruberman)

\begin{abstract}
We determine the structure of the first resonance variety of the cohomology ring of the group of automorphisms of a finitely generated free group which act by conjugation on a given basis.
\end{abstract}

\section{Resonance of $P \Sigma_{n}$}

Let $F_{n}$ be the free group generated by $x_{1}, \ldots, x_{n}$. The basis-conjugating automorphism group, or pure symmetric automorphism group, is the group $P \Sigma_{n}$ of all automorphisms of $F_{n}$ which send each generator $x_{i}$ to a conjugate of itself. Results of Dahm [4] and Goldsmith [7] imply that this group may also be realized as the "group of loops", the group of motions of a collection of $n$ unknotted, unlinked oriented circles in 3-space, where each circle returns to its original position. McCool 9] found the following presentation for the basis-conjugating automorphism group:

$$
P \Sigma_{n}=\left\langle\beta_{i, j}, 1 \leq i \neq j \leq n \mid\left[\beta_{i, j}, \beta_{k, l}\right],\left[\beta_{i, k}, \beta_{j, k}\right],\left[\beta_{i, j},\left(\beta_{i, k} \cdot \beta_{j, k}\right)\right]\right\rangle,
$$

where $[u, v]=u v u^{-1} v^{-1}$ denotes the commutator, the indices in the relations are distinct, and the generators $\beta_{i, j}$ are the automorphisms of $F_{n}$ defined by

$$
\beta_{i, j}\left(x_{k}\right)= \begin{cases}x_{k} & \text { if } k \neq j, \\ x_{j}^{-1} x_{i} x_{j} & \text { if } k=i .\end{cases}
$$

The purpose of this paper is to determine the structure of the first resonance variety of the cohomology ring of this group.

Let $A=\bigoplus_{k=0}^{\ell} A^{k}$ be a finite-dimensional, graded, connected algebra over an algebraically closed field $\mathbb{k}$ of characteristic 0 . Since $a \cdot a=0$ for each $a \in A^{1}$, multiplication by $a$ defines a cochain complex $\left(A, \delta_{a}\right)$ :

$$
A^{0} \stackrel{\delta_{a}}{\longrightarrow} A^{1} \stackrel{\delta_{a}}{\longrightarrow} A^{2} \stackrel{\delta_{a}}{\longrightarrow} \cdots \cdots \stackrel{\delta_{a}}{\longrightarrow} A^{\ell},
$$

where $\delta_{a}(x)=a x$. The resonance varieties of $A$ are the jumping loci for the cohomology of these complexes: $R_{d}^{j}(A)=\left\{a \in A^{1} \mid \operatorname{dim}_{\mathbb{k}} H^{j}\left(A, \delta_{a}\right) \geq d\right\}$. As shown by Falk 6, these algebraic subvarieties of $A^{1}$ are isomorphism-type invariants of the algebra $A$. They have been the subject of considerable recent interest in the context of hyperplane arrangements and related areas; see, for instance, Dimca,

Received by the editors November 17, 2008.

2000 Mathematics Subject Classification. Primary 20F28; Secondary 14M12, 20J06.

The author was partially supported by National Security Agency grant No. H98230-05-1-0055.

(C)2009 American Mathematical Society Reverts to public domain 28 years from publication 
Papadima, and Suciu [5], Yuzvinsky [14, and the references therein. We will focus on the first resonance variety $R^{1}(A)=\left\{a \in A^{1} \mid H^{1}\left(A, \delta_{a}\right) \neq 0\right\}$.

Since the relations in the presentation (1.1) of $P \Sigma_{n}$ are all commutators, the first homology group $H_{1}\left(P \Sigma_{n} ; \mathbb{k}\right)$ is a vector space of dimension $n(n-1)$ with basis $\left\{\left[\beta_{p, q}\right] \mid 1 \leq p \neq q \leq \ell\right\}$. Let $\left\{e_{p, q} \mid 1 \leq p \neq q \leq \ell\right\}$ be the dual basis of $H^{1}\left(P \Sigma_{n} ; \mathbb{k}\right)$. Denote the first resonance variety of $A=H^{*}\left(P \Sigma_{n} ; \mathbb{k}\right)$ by $R^{1}\left(P \Sigma_{n}, \mathbb{k}\right)$.

Theorem 1.1. The first resonance variety of the cohomology $\operatorname{ring} H^{*}\left(P \Sigma_{n} ; \mathbb{k}\right)$ of the basis-conjugating automorphism group is

$$
R^{1}\left(P \Sigma_{n}, \mathbb{k}\right)=\bigcup_{1 \leq i<j \leq n} C_{i, j} \cup \bigcup_{1 \leq i<j<k \leq n} C_{i, j, k},
$$

where $C_{i, j}=\operatorname{span}\left\{e_{i, j}, e_{j, i}\right\}$ and $C_{i, j, k}=\operatorname{span}\left\{e_{j, i}-e_{k, i}, e_{i, j}-e_{k, j}, e_{i, k}-e_{j, k}\right\}$.

This result reveals an interesting relationship between the resonance variety and the Bieri-Neumann-Strebel (BNS) invariant of the basis-conjugating automorphism group. For a finitely generated group $G$, let $\mathcal{C}$ be the Cayley graph corresponding to a finite generating set. Given an additive character $\chi: G \rightarrow \mathbb{R}$, let $\mathcal{C}_{+}(\chi)$ be the full subgraph of $\mathcal{C}$ on the vertex set $\{g \in G \mid \chi(g) \geq 0\}$. Then the (first) BNS invariant of $G$ is the conical subset $\Sigma(G)$ of $\operatorname{Hom}(G, \mathbb{R})=H^{1}(G ; \mathbb{R})$ defined by

$$
\Sigma(G)=\left\{\chi \in \operatorname{Hom}(G, \mathbb{R}) \backslash\{0\} \mid \mathcal{C}_{+}(\chi) \text { is connected }\right\} .
$$

This invariant of $G$ (which is independent of the choice of generating set) may be used to determine which subgroups above the commutator subgroup $[G, G]$ are finitely generated; see [1].

The BNS invariant of the group $G=P \Sigma_{n}$ was determined by Orlandi-Korner 11. Combining her result with the above theorem yields the following:

Theorem 1.2. The Bieri-Neumann-Strebel invariant of the basis-conjugating automorphism group is given by

$$
\Sigma\left(P \Sigma_{n}\right)=H^{1}\left(P \Sigma_{n} ; \mathbb{R}\right) \backslash R^{1}\left(P \Sigma_{n}, \mathbb{R}\right) .
$$

This relationship between the resonance variety and the BNS invariant is known to hold for certain other groups, including right-angled Artin groups; see Meier and VanWyk [10] and Papadima and Suciu [12. More recently, Papadima and Suciu have shown that the containment $\Sigma(G) \subseteq H^{1}(G ; \mathbb{R}) \backslash R^{1}(G, \mathbb{R})$ holds for an arbitrary 1-formal group $G$, where, as above, $R^{1}(G ; \mathbb{R})$ denotes the first resonance variety of $H^{*}(G ; \mathbb{R})$. See $[13$, for a detailed investigation of the relationship between BNS invariants and (co)homology jumping loci in a number of contexts.

\section{Proof of Theorem 1.1}

In this section, we recall the structure of the cohomology ring of the basisconjugating automorphism group and use it to prove Theorem 1.1. The cohomology of $P \Sigma_{n}$ was computed by Jensen, McCammond, and Meier 8 , resolving positively a conjecture of Brownstein and Lee [2].

Theorem $2.1([8])$. Let $E_{\mathbb{Z}}$ denote the exterior algebra over $\mathbb{Z}$ generated by degree one elements $e_{p, q}, 1 \leq p \neq q \leq n$, and let $I_{\mathbb{Z}}$ denote the two-sided ideal in $E_{\mathbb{Z}}$ generated by

$$
\begin{aligned}
\eta_{i, j} & =e_{i, j} e_{j, i}, 1 \leq i<j \leq n \\
\tau_{i, j}^{k} & =\left(e_{k, i}-e_{j, i}\right)\left(e_{k, j}-e_{i, j}\right), 1 \leq k \leq n, 1 \leq i<j \leq n, k \notin\{i, j\} .
\end{aligned}
$$


Then the integral cohomology algebra of the basis-conjugating automorphism group $P \Sigma_{n}$ is isomorphic to the quotient of $E_{\mathbb{Z}}$ by $I_{\mathbb{Z}}, H^{*}\left(P \Sigma_{n} ; \mathbb{Z}\right) \cong E_{\mathbb{Z}} / I_{\mathbb{Z}}$.

Remark 2.2. The above presentation of the cohomology ring $H^{*}\left(P \Sigma_{n} ; \mathbb{Z}\right)$ differs slightly from that given in [2, 8, but is easily seen to be equivalent. For instance, the relation in $H^{*}\left(P \Sigma_{n} ; \mathbb{Z}\right)$ arising from the generator $\tau_{i, j}^{k}$ of the ideal $I$ may be obtained by constructing an appropriate linear combination of the relations labeled 2 and 3 in [8, Thm. 6.7].

Let $\mathbb{k}$ be a field of characteristic zero. From Theorem 2.1 and the Universal Coefficient Theorem, the cohomology algebra $H^{*}\left(P \Sigma_{n} ; \mathbb{k}\right)$ is isomorphic to $E_{\mathbb{k}} / I_{\mathbb{k}}$, where $E_{\mathbb{k}}$ is the exterior algebra over $\mathbb{k}$ generated by $e_{p, q}, 1 \leq p \neq q \leq n$, and $I_{\mathbb{k}}$ is the ideal in $E_{\mathrm{k}}$ generated by the elements $\eta_{i, j}$ and $\tau_{i, j}^{k}$ above.

Recall from Section 1 that the first resonance variety of $A=H^{*}\left(P \Sigma_{n} ; \mathbb{k}\right)$ is

$$
R^{1}\left(P \Sigma_{n}, \mathbb{k}\right)=\left\{a \in A^{1} \mid H^{1}\left(A, \delta_{a}\right) \neq 0\right\} .
$$

Observe that $A^{1}=H^{1}\left(P \Sigma_{n} ; \mathbb{k}\right)$ is a vector space of dimension $N=n(n-1)$ over $\mathbb{k}$. Elements of $A^{1}$ are of the form $a=\sum_{p \neq q} a_{p, q} e_{p, q}$, where $a_{p, q} \in \mathbb{k}$. Recall that

$$
C_{i, j}=\operatorname{span}\left\{e_{i, j}, e_{j, i}\right\}=\left\{a \in A^{1} \mid a_{p, q}=0 \text { if }\{p, q\} \neq\{i, j\}\right\}
$$

for $1 \leq i<j \leq n$ and that

$$
\begin{aligned}
C_{i, j, k} & =\operatorname{span}\left\{e_{j, i}-e_{k, i}, e_{i, j}-e_{k, j}, e_{i, k}-e_{j, k}\right\} \\
& =\left\{\begin{array}{l|l}
a \in A^{1} & \begin{array}{l}
a_{j, i}+a_{k, i}=0, a_{i, j}+a_{k, j}=0, a_{i, k}+a_{j, k}=0, \\
a_{p, q}=0 \text { if }\{p, q\} \not \subset\{i, j, k\}
\end{array}
\end{array}\right\}
\end{aligned}
$$

for $1 \leq i<j<k \leq n$. Note that these are linear subspaces of $A^{1} \cong \mathbb{k}^{N}$ of dimensions 2 and 3 respectively. To prove Theorem 1.1 we must show that $R^{1}\left(P \Sigma_{n}, \mathbb{k}\right)$ is equal to the union of these linear subspaces.

Proof of Theorem 1.1. In the case $n=2$, the group $P \Sigma_{n}=\left\langle\beta_{1,2}, \beta_{2,1}\right\rangle \cong F_{2}$ is a free group, and the theorem asserts that $R^{1}\left(P \Sigma_{2}, \mathbb{k}\right)=C_{1,2}=H^{1}\left(P \Sigma_{2} ; \mathbb{k}\right)$, which is clear. So assume that $n \geq 3$.

Write $R=R^{1}\left(P \Sigma_{n}, \mathbb{k}\right)$ and $C=\bigcup_{1 \leq i<j \leq n} C_{i, j} \cup \bigcup_{1 \leq i<j<k \leq n} C_{i, j, k}$. Observe that $0 \in C$ and $0 \in R$. So it is enough to show that $R \backslash\{0\}=C \backslash\{0\}$.

Write $E=E_{\mathbb{k}}$ and $I=I_{\mathbb{k}}$. For $a \in A^{1}=E^{1}$, we have a short exact sequence of chain complexes $0 \longrightarrow\left(I, \delta_{a}\right) \stackrel{\iota}{\longrightarrow}\left(E, \delta_{a}\right) \stackrel{p}{\longrightarrow}\left(A, \delta_{a}\right) \longrightarrow 0$ :

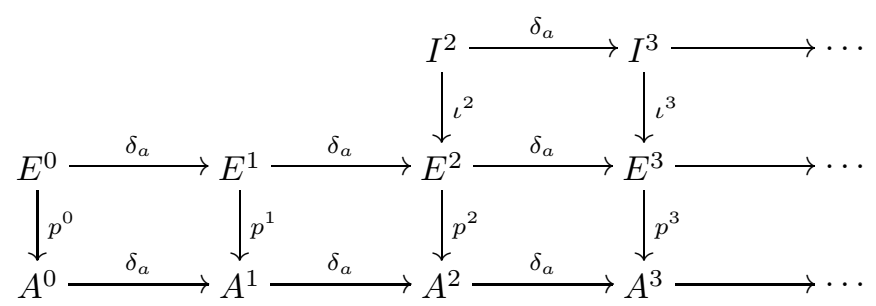

where $\iota: I \rightarrow E$ is the inclusion, $p: E \rightarrow A$ the projection, and $\delta_{a}(x)=a x$. Note that since $I$ is generated in degree two, the maps $p^{0}: E^{0} \rightarrow A^{0}$ and $p^{1}: E^{1} \rightarrow A^{1}$ are identity maps. If $a \neq 0$, then the complex $\left(E, \delta_{a}\right)$ is acyclic. Consequently, the corresponding long exact cohomology sequence yields

$$
H^{1}\left(A, \delta_{a}\right) \cong H^{2}\left(I, \delta_{a}\right)=\operatorname{ker}\left(\delta_{a}: I^{2} \rightarrow I^{3}\right)=\operatorname{ker}\left(\iota^{3} \circ \delta_{a}: I^{2} \rightarrow E^{3}\right) .
$$


Thus, $a \in R \backslash\{0\}$ if and only if the map $\psi_{a}:=\iota^{3} \circ \delta_{a}$ fails to inject.

Since the elements $\eta_{i, j}$ and $\tau_{i, j}^{k}$ recorded in (2.1) generate the ideal $I$ and are of degree two (and are linearly independent in $E^{2}$ ), these elements form a basis for $I^{2}$. We record the images of these basis elements under the map $\psi_{a}$. For $1 \leq i<j \leq n$,

$$
\psi_{a}\left(\eta_{i, j}\right)=\sum_{\{p, q\} \neq\{i, j\}} a_{p, q} e_{p, q} \eta_{i, j}=\sum_{\{p, q\} \neq\{i, j\}} a_{p, q} e_{p, q} e_{i, j} e_{j, i}
$$

For $1 \leq k \leq n, 1 \leq i<j \leq n, k \notin\{i, j\}$,

$$
\begin{gathered}
\psi_{a}\left(\tau_{i, j}^{k}\right)=\left(a_{j, i}+a_{k, i}\right) e_{j, i} e_{k, i}\left(e_{k, j}-e_{i, j}\right)-\left(a_{i, j}+a_{k, j}\right) e_{i, j} e_{k, j}\left(e_{k, i}-e_{j, i}\right) \\
+\left(a_{i, k} e_{i, k}+a_{j, k} e_{j, k}\right) \tau_{i, j}^{k}+\sum_{\{p, q\} \not \subset\{i, j, k\}} a_{p, q} e_{p, q} \tau_{i, j}^{k} .
\end{gathered}
$$

These calculations immediately yield the containment $C \backslash\{0\} \subseteq R \backslash\{0\}$. If $a \in C_{i, j}$, then $a_{p, q}=0$ for $\{p, q\} \neq\{i, j\}$. For such an $a$, we have $\psi_{a}\left(\eta_{i, j}\right)=0$ by (2.4), so $C_{i, j} \subset R$. If $1 \leq i<j<k \leq n$ and $a \in C_{i, j, k}$, then $a_{j, i}+a_{k, i}=0$, $a_{i, j}+a_{k, j}=0, a_{i, k}+a_{j, k}=0$, and $a_{p, q}=0$ for $\{p, q\} \not \subset\{i, j, k\}$. In this instance, (2.5) may be used to check that $a_{j, i} \tau_{i, j}^{k}-a_{i, k} \tau_{j, k}^{i}$ and $a_{i, j} \tau_{i, j}^{k}-a_{i, k} \tau_{i, k}^{j}$ are elements of $\operatorname{ker}\left(\psi_{a}\right)$. If $a \in C_{i, j, k}$ is nonzero, at least one of $a_{i, j}, a_{i, k}, a_{j, i}$ must be nonzero. Consequently, $\psi_{a}$ has nontrivial kernel, and $C_{i, j, k} \subset R$.

Establishing the reverse containment, $R \backslash\{0\} \subseteq C \backslash\{0\}$, is more involved. We will show that $a \notin C$ implies that $a \notin R$. If $a \notin C$, then $a \neq 0$. So assume without loss that $a_{2,1} \neq 0$. Since $a \notin C_{1,2} \subset C$, we must also have $a_{p, q} \neq 0$ for some $\{p, q\} \neq\{1,2\}$. We will consider several cases depending on the relationship between the sets $\{1,2\}$ and $\{p, q\}$.

Case 1. $\{1,2\} \cap\{p, q\}=\emptyset$

Assume first that $\{1,2\}$ and $\{p, q\}$ are disjoint. Note that $n \geq 4$ in this instance. Permuting indices if need be, we may assume that $a \in H^{1}\left(P \Sigma_{n} ; \mathbb{k}\right)$ satisfies $a_{2,1} \neq 0$ and $a_{3,4} \neq 0$. We will show that this assumption implies that the map $\psi_{a}: I^{2} \rightarrow E^{3}$ injects; hence $a \notin R$. Specifically, we will exhibit a subspace $V \subset E^{3}$ and a projection $\pi: E^{3} \rightarrow V$ so that the composition $\pi \circ \psi_{a}: I^{2} \rightarrow V$ is an isomorphism.

Let $\mathcal{V}$ be the union of the sets

$$
\begin{aligned}
& \left\{e_{1,2} e_{2,1} e_{3,4}\right\} \cup\left\{e_{2,1} e_{i, j} e_{j, i} \mid 1 \leq i<j \leq n,\{i, j\} \neq\{1,2\}\right\}, \\
& \left\{e_{3,4} e_{1,2} e_{k, 1}, e_{3,4} e_{2,1} e_{1, k}, e_{3,4} e_{1,2} e_{1, k} \mid 3 \leq k \leq n\right\}, \\
& \left\{e_{2,1} e_{k, i} e_{k, j}, e_{2,1} e_{j, i} e_{j, k}, e_{2,1} e_{i, k} e_{k, j} \mid i \leq 2<j<k \leq n \text { or } 3 \leq i<j<k \leq n\right\} .
\end{aligned}
$$

There is a bijection between $\mathcal{V}$ and the set of generators of $I^{2}$ given by

$$
\begin{aligned}
& \eta_{1,2} \leftrightarrow e_{1,2} e_{2,1} e_{3,4}, \eta_{i, j} \leftrightarrow e_{2,1} e_{i, j} e_{j, i} \quad(\{i, j\} \neq\{1,2\}), \\
& \tau_{1,2}^{k} \leftrightarrow e_{3,4} e_{1,2} e_{k, 1}, \quad \tau_{1, k}^{2} \leftrightarrow e_{3,4} e_{2,1} e_{1, k}, \quad \tau_{2, k}^{1} \leftrightarrow e_{3,4} e_{1,2} e_{1, k} \quad(3 \leq k \leq n), \\
& \tau_{i, j}^{k} \leftrightarrow e_{2,1} e_{k, i} e_{k, j}, \quad \tau_{i, k}^{j} \leftrightarrow e_{2,1} e_{j, i} e_{j, k}, \quad \tau_{j, k}^{i} \leftrightarrow e_{2,1} e_{i, k} e_{k, j} \quad(i \leq 2<j<k \leq n) .
\end{aligned}
$$

In particular, the monomials in $\mathcal{V}$ are distinct. Hence, $\mathcal{V}$ is a linearly independent set in $E^{3}$ of cardinality $|\mathcal{V}|=\operatorname{dim}_{\mathbb{k}} I^{2}=\left(\begin{array}{l}n \\ 2\end{array}\right)(n-1)$. Let $V=\operatorname{span} \mathcal{V} \subset E^{3}$.

Define $\pi: E^{3} \rightarrow V$ on basis elements by

$$
\pi\left(e_{a, b} e_{c, d} e_{p, q}\right)= \begin{cases}e_{a, b} e_{c, d} e_{p, q} & \text { if } e_{a, b} e_{c, d} e_{p, q} \in \mathcal{V}, \\ 0 & \text { if } e_{a, b} e_{c, d} e_{p, q} \notin \mathcal{V}\end{cases}
$$


Then, a calculation using (2.4) and (2.5) reveals that $\pi \circ \psi_{a}: I^{2} \rightarrow V$ is an isomorphism. For instance, ordering the bases of $I^{2}$ and $V$ appropriately, one can check that the matrix $\mathrm{M}$ of $\pi \circ \psi_{a}$ has determinant $\operatorname{det} \mathrm{M}=y_{2,1}^{m_{2,1}} y_{3,4}^{m_{3,4}} \neq 0$, where $m_{3,4}=3 n-5$ and $m_{2,1}=\left(\begin{array}{c}n \\ 2\end{array}\right)(n-1)-m_{3,4}$. Thus, if $a \notin C$ satisfies $a_{2,1} \neq 0$ and $a_{p, q} \neq 0$ for some $p, q$ with $\{1,2\} \cap\{p, q\}=\emptyset$, then $a \notin R$.

Case 2. $\{1,2\} \cap\{p, q\} \neq \emptyset$

Now assume that $a \notin C, a_{2,1} \neq 0$, and $a_{r, s}=0$ for all $r, s$ with $\{1,2\} \cap\{r, s\}=\emptyset$. Since $a \notin C_{1,2} \subset C$, we must have $a_{p, q} \neq 0$ for some $p, q$ with $|\{p, q\} \cap\{1,2\}|=1$. Permuting indices if necessary, we may assume that $3 \in\{p, q\}$.

In the case $n=3$, since $a \notin C_{1,2,3} \subset C$, one of the sums $a_{2,1}+a_{3,1}, a_{1,2}+a_{3,2}$, $a_{1,3}+a_{2,3}$ must be nonzero; see (2.3). In this instance, ordering bases appropriately, the map $\psi_{a}: I^{2} \rightarrow E^{3}$ has matrix

$$
\mathbf{M}_{3}=\left(\begin{array}{cccccc}
a_{3,2} & 0 & 0 & 0 & -a_{1,2}-a_{3,2} & 0 \\
a_{3,1} & 0 & 0 & 0 & -a_{2,1}-a_{3,1} & 0 \\
a_{2,3} & 0 & 0 & a_{1,2} & -a_{2,3} & a_{2,1} \\
-a_{1,3} & 0 & 0 & a_{1,2} & a_{1,3} & a_{2,1} \\
0 & a_{3,2} & 0 & -a_{3,2} & a_{1,3} & -a_{3,1} \\
0 & -a_{2,3} & 0 & a_{1,3}+a_{2,3} & 0 & 0 \\
0 & -a_{2,1} & 0 & a_{2,1}+a_{3,1} & 0 & 0 \\
0 & a_{1,2} & 0 & -a_{1,2} & -a_{1,3} & a_{3,1} \\
0 & 0 & -a_{3,1} & a_{3,2} & a_{2,3} & a_{3,1} \\
0 & 0 & a_{2,1} & a_{3,2} & a_{2,3} & -a_{2,1} \\
0 & 0 & a_{1,3} & 0 & 0 & -a_{1,3}-a_{2,3} \\
0 & 0 & a_{1,2} & 0 & 0 & -a_{1,2}-a_{3,2} \\
0 & 0 & 0 & a_{3,2} & -a_{1,3} & -a_{2,1} \\
0 & 0 & 0 & a_{2,1}+a_{3,1} & 0 & 0 \\
0 & 0 & 0 & a_{1,3}+a_{2,3} & 0 & 0 \\
0 & 0 & 0 & a_{1,2} & -a_{2,3} & -a_{3,1} \\
0 & 0 & 0 & 0 & a_{1,2}+a_{3,2} & 0 \\
0 & 0 & 0 & 0 & a_{2,1}+a_{3,1} & 0 \\
0 & 0 & 0 & 0 & 0 & a_{1,2}+a_{3,2} \\
0 & 0 & 0 & 0 & 0 & a_{1,3}+a_{2,3}
\end{array}\right)
$$

Using the assumptions on $a_{2,1}$, the sums $a_{2,1}+a_{3,1}, a_{1,2}+a_{3,2}, a_{1,3}+a_{2,3}$, and $a_{p, q} \in\left\{a_{1,3}, a_{2,3}, a_{3,1}, a_{3,2}\right\}$, one can check that the matrix $\mathrm{M}_{3}$ has maximal rank. Hence, if $a \notin C$, then $\psi_{a}: I^{2} \rightarrow E^{3}$ injects in the case $n=3$.

For general $n$, the assumption that $a \notin C_{1,2,3}$ implies that the set

$$
\left\{a_{2,1}+a_{3,1}, a_{1,2}+a_{3,2}, a_{1,3}+a_{2,3}\right\} \cup\left\{a_{r, s} \mid\{r, s\} \not \subset\{1,2,3\}\right\}
$$

contains a nonzero element. Recall that, by Case 1 , we may assume that $a_{r, s}=0$ for all $r, s$ with $\{1,2\} \cap\{r, s\}=\emptyset$. If $a_{r, s}=0$ for all $\{r, s\} \not \subset\{1,2,3\}$, let $W$ be the subspace of $E^{3}$ spanned by the union of the sets

$$
\begin{aligned}
& \left\{e_{i_{1}, j_{1}} e_{i_{2}, j_{2}} e_{i_{3}, j_{3}} \mid 1 \leq i_{k}, j_{k} \leq 3, i_{k} \neq j_{k}\right\} \text {, } \\
& \left\{e_{1,2} e_{2,1} e_{p, q}\right\} \cup\left\{e_{2,1} e_{i, j} e_{j, i} \mid 1 \leq i<j \leq n,\{i, j\} \neq\{1,2\}\right\}, \\
& \left\{e_{2,1} e_{k, i} e_{k, j}, e_{2,1} e_{j, i} e_{j, k}, e_{2,1} e_{i, k} e_{k, j} \mid i \leq 2<j<k \leq n \text { or } 3 \leq i<j<k \leq n\right\} \text {, } \\
& \left\{\begin{array}{lll}
e_{p, q} e_{k, 1} e_{k, 2}, & e_{p, q} e_{k, 1} e_{1,2}, & e_{p, q} e_{2,1} e_{k, 2}, \\
e_{p, q} e_{2,1} e_{2, k}, & e_{p, q} e_{2,1} e_{1, k}, & e_{p, q} e_{k, 1} e_{2, k}, \\
e_{p, q} e_{1,2} e_{1, k}, & e_{p, q} e_{1,2} e_{2, k}, & e_{p, q} e_{k, 2} e_{1, k}
\end{array} \mid 3 \leq k \leq n\right\} .
\end{aligned}
$$


Define $\pi: E^{3} \rightarrow W$ on basis elements as before. Ordering bases appropriately, one can use (2.4) and (2.5) to find a submatrix $\mathrm{M}$ of the matrix of $\pi \circ \psi_{a}: I^{2} \rightarrow W$ of the form

$$
\mathrm{M}=\left(\begin{array}{cc}
\mathrm{U} & * \\
0 & \mathrm{M}_{3}
\end{array}\right)
$$

where $\mathrm{M}_{3}$ is given by (2.6) and $\mathrm{U}$ is upper triangular, with diagonal entries $a_{2,1} \neq 0$ and $a_{p, q} \neq 0$. (The choice of $\mathrm{U}$ depends on which $a_{p, q} \in\left\{a_{1,3}, a_{2,3}, a_{3,1}, a_{3,2}\right\}$ is nonzero.) Hence, the matrix $\mathrm{M}$ has maximal rank. It follows that $\psi_{a}: I^{2} \rightarrow E^{3}$ injects.

Finally, consider the case where $a \notin C_{1,2,3} \subset C, a_{2,1} \neq 0, a_{p, q} \neq 0$ for some $a_{p, q} \in\left\{a_{1,3}, a_{2,3}, a_{3,1}, a_{3,2}\right\}$, and $a_{r, s} \neq 0$ for some $\{r, s\} \not \subset\{1,2,3\}$. Since we may assume by Case 1 that $a_{r, s}=0$ if $\{1,2\} \cap\{r, s\}=\emptyset$, we have $a_{r, s} \neq 0$ for some $r, s$ with $r \in\{1,2\}$ and $4 \leq s \leq n$. In this instance, let $W$ be the subspace of $E^{3}$ spanned by the union of the sets

$$
\begin{aligned}
& \left\{e_{1,2} e_{2,1} e_{p, q}\right\} \cup\left\{e_{2,1} e_{i, j} e_{j, i} \mid 1 \leq i<j \leq n,\{i, j\} \neq\{1,2\}\right\}, \\
& \left\{e_{2,1} e_{k, i} e_{k, j}, e_{2,1} e_{j, i} e_{j, k}, e_{2,1} e_{i, k} e_{k, j} \mid i \leq 2<j<k \leq n \text { or } 3 \leq i<j<k \leq n\right\}, \\
& \left\{e_{r, s} e_{3,1} e_{3,2}, e_{r, s} e_{1,3} e_{3,2}, e_{r, s} e_{2,3} e_{3,1}\right\}, \\
& \left\{e_{p, q} e_{k, 1} e_{k, 2}, e_{p, q} e_{2, k} e_{k, 1}, e_{p, q} e_{1, k} e_{k, 2} \mid 4 \leq k \leq n\right\} .
\end{aligned}
$$

Defining $\pi: E^{3} \rightarrow W$ on basis elements as above, a calculation using (2.4) and (2.5) shows that $\pi \circ \psi_{a}: I^{2} \rightarrow W$ is an isomorphism. For instance, ordering bases appropriately, one can check that the matrix $\mathrm{M}$ of $\pi \circ \psi_{a}$ has determinant $\operatorname{det} \mathrm{M}=$ $a_{2,1}^{m_{2,1}} a_{p, q}^{m_{p, q}} a_{r, s}^{3}$, where $m_{p, q}=3 n-8$ and $m_{2,1}=\left(\begin{array}{c}n \\ 2\end{array}\right)(n-1)-m_{p, q}$. Hence, $\psi_{a}$ injects in this final case.

Thus, for any $a \notin C$, the map $\psi_{a}: I^{2} \rightarrow E^{3}$ injects, and $a \notin R$. This completes the proof of Theorem 1.1

Remark 2.3. It follows from Theorem 2.1 that the integral cohomology groups of $P \Sigma_{n}$ are torsion free, with Betti numbers $b_{k}\left(P \Sigma_{n}\right)=\operatorname{rank} H^{k}\left(P \Sigma_{n} ; \mathbb{Z}\right)$ given by the coefficients of the Poincaré polynomial $\mathfrak{p}\left(P \Sigma_{n}, t\right)=\sum_{k>0} b_{k}\left(P \Sigma_{n}\right) \cdot t^{k}=(1+n t)^{n-1}$; see $[8, \S 6]$. Thus the cohomology groups cannot distinguish $P \Sigma_{n}$ from a direct product $F_{n} \times \cdots \times F_{n}$ of $n-1$ free groups of rank $n$. For $n=2$, this is to be expected, since $P \Sigma_{2} \cong F_{2}$.

For $n \geq 3$, the groups $P \Sigma_{n}$ and $F_{n} \times \cdots \times F_{n}$ are not isomorphic and are, in fact, distinguished by their cohomology rings. By Theorem 1.1, the irreducible components of $R^{1}\left(P \Sigma_{n}, \mathbb{k}\right)$ are two- and three-dimensional. On the other hand, the results of [3] or [12] may be used to show that the irreducible components of the first resonance variety of $H^{*}\left(F_{n} \times \cdots \times F_{n} ; \mathbb{k}\right)$ are all $n$-dimensional.

\section{ACKNOWLEDGMENTS}

We thank Graham Denham and Michael Falk for useful conversations.

\section{REFERENCES}

1. R. Bieri, W. D. Neumann, R. Strebel, A geometric invariant of discrete groups, Invent. Math. 90 (1987), 451-477. MR0914846

2. A. Brownstein, R. Lee, Cohomology of the group of motions of $n$ strings in 3-space, in: Mapping class groups and moduli spaces of Riemann surfaces (Goettingen, 1991 / Seattle, WA, 1991), pp. 51-61, Contemp. Math. 150, Amer. Math. Soc., Providence, RI, 1993. MR.1234259 
3. D. Cohen, A. Suciu, Characteristic varieties of arrangements, Math. Proc. Cambridge Phil. Soc. 127 (1999), 33-53. MR.1692519

4. D. Dahm, A generalization of a braid theory, Ph.D. Thesis, Princeton Univ., 1962.

5. A. Dimca, S. Papadima, A. Suciu, Topology and geometry of cohomology jumping loci, Duke Math. J., to appear. arXiv:0902.1250

6. M. Falk, Arrangements and cohomology, Ann. Combin. 1 (1997), 135-157. MR1629681

7. D. Goldsmith, The theory of motion groups, Michigan Math. J. 28 (1981), 3-17. MR0600411

8. C. Jensen, J. McCammond, J. Meier, The integral cohomology of the group of loops, Geom. Topol. 10 (2006), 759-784. MR2240905

9. J. McCool, On basis-conjugating automorphisms of free groups, Canad. J. Math. 38 (1986), 1525-1529. MR0873421

10. J. Meier, L. VanWyk, The Bieri-Neumann-Strebel invariants for graph groups, Proc. London Math. Soc. (3) 71 (1995), 263-280. MR1337468

11. L. Orlandi-Korner, The Bieri-Neumann-Strebel invariant for basis-conjugating automorphisms of free groups, Proc. Amer. Math. Soc. 128 (2000), 1257-1262. MR1712889

12. S. Papadima, A. Suciu, Algebraic invariants for right-angled Artin groups, Math. Ann. 334 (2006), 533-555. MR2207874

13. S. Papadima, A. Suciu, Bieri-Neumann-Strebel-Renz invariants and homology jumping loci, preprint, 2008. arXiv:0812.2660

14. S. Yuzvinsky, Orlik-Solomon algebras in algebra and topology, Russian Math. Surveys 56 (2001), 293-364. MR1859708

Department of Mathematics, Louisiana State University, Baton Rouge, Louisiana 70803

E-mail address: cohen@math.lsu.edu

URL: http://www.math.1su.edu/ cohen/ 